che des streptocoques dans l'expertise du lait. Het Algemmen Zuivelblad. 1922, 2, 28. Repris de Het Chemisch Weekblad. An. in Le Lait.

[14] A. A. Veenbaas. L'examen du sédiment du lait, spécialement au point de vue de la signification des streptocoques dans l'appréciation du lait. Het Chemisch Weekblad, 1922, 19, 283. An. in Le Lait.

[15] C. Gorint. Action des streptocoques dans le lait. Comptes rendus de l'Académie des Sciences. An, in Le Lait.

[16] Davis et Matrick. Rusty Spot in Cheddar and other Cheese. I. Description of the causative organism. The Journal of Dairy Research. 1929, 1, 1, 50.

[17] Orla Jensen. The Lactic Acid Bacteria, 1919, Copenhague.

[18] Davis et Matrick. Rusty Spot in Cheddar and other cheese. II. Factors controlling the Pigmentation of the causative organism. The Journal of Dairy Research, 1930, 1, 2, 135 .

\title{
LE FERMENT DE LA PRÉSURE ET LE LAIT (1)
}

par

\section{RENÉ GALLAY}

Le phénomène banal, à portée de tout le monde, du lait qui tranche, qui caille, qui coagule sous l'action d'un peu de présure, a été l'objet de nombreuses recherches. Il existe sur cette question qui intéresse tout en même temps le physiologiste, puisque le phénomène se produit dans l'estomac et participe à la digestion, le chimiste, parce qu'il est important de savoir quelles sont les modifications moléculaires qui l'accompagnent, et le technicien, qui est forcé d'y voir la transformation du lait se trouvant à l'origine de l'industrie fromagère, une littérature volumineuse, encombrante, souvent touffue, regorgeant d'explications contradictoires, et dont la fantaisie n'est pas toujours exclue. Le but que nous nous sommes proposés aujourd'hui est de dégager de ce fatras de connaissances, de chiffres et d'hypothèses, les connaissances qui sont le plus solidement étayées sur l'expérimentation et, en nous aidant parfois d'observations et de recherehes personnelles, tenter de leur donner leur signification réelle, pour répondre finalement à ces trois questions : qu'est-ce que la présure ? quel est le mécanisme de son action chimique sur le lait ? et pourquoi conduit-elle à la prise en masse de ce liquide, e'est-à-dire à la solidification d'une partie de ses constituants ?

Le ferment soluble, l'enzyme de la présure, capable, sous certaines conditions, de trancher le lait, est produit par l'estomac de tous les mammifères, mais en quantité fort variable. On le connaît

(1) Leçon inaugurale d'un cours de chimie du lait, prononcée le 25 avril 1934 à l'Université de Lausanne. 
encore sous les noms de chymosine, chymase ou lab. Quelques expérimentateurs, faisant porter leurs recherches sur d'autres classes de vertébrés, ont extrait du tube digestif des oiseaux et des poissons certaines substances pouvant trancher le lait, auxquelles ils ont donné le nom de présure, sans cependant fournir la preuve que ces matières présentent avec celles qui sont sécrétées par les estomacs de mammifères, des caractères de similitude chimique. C'est aussi sons le nom de présure qu'on a désigné les substances d'origine végétale contenues dans la sève de certaines plantes, comme le figuier, l'artichaut, dont l'homme s'est servi longtemps pour coaguler le lait et préparer divers fromages. Nous aurons montré la très grande dispersion de ce ferment de la présure si nous ajoutons enfin qu'il est sécrété encore par de nombreuses espèces microbiennes.

Si l'idée de prolonger la durée de conservation du lait en en préparant du fromage se perd dans la nuit des temps, l'utilisation de la présure dans ce but est certainement moins ancienne. Elle est fort vieille cependant. Quelque mille ans avant Jésus-Christ, les Grecs s'en servaient déjà. Sous les Romains, la fabrication des fromages à présures à l'aide du lait tranché extrait de l'estomac de jeunes ruminants, connut un développement très généralisé. Le caseus helveticus, déjà réputé, était vraisemblablement produit par une méthode analogue. Quant aux Germains, - signe évident de leur manque de civilisation, - ils ne semblaient connaître pour trancher le lait, jusqu'au moment où ils s'approprièrent les connaissances de leurs vaincus, que le procédé archaïque de l'acidification naturelle.

Les recherches par lesquelles physiologistes et chimistes ont abordé l'étude des propriétés de la présure et se sont efforcés de définir la composition chimique de ce ferment ont donné naissance à deux conceptions, représentées par deux écoles. La première, mais non point la plus ancienne, affirme avec PAWLOW, BANG et d'autres que la distinction faite ordinairement entre présure et pepsine est purement arbitraire, spécieuse, ne repose-sur aucun fondement solide et qu'il ne s'agit en réalité que d'un seul ferment agissant tantôt d'une façon comme coagulant, tantôt d'une autre comme solubilisant. C'est le point de vue uniciste, qu'il y a peu d'années encore, ralliait de nombreux partisans. En d'autres termes, pepsine et présure seraient simplement deux aspects d'un ferment identique mais dont le mode d'action serait conditionné essentiellement par la réaction de milieu, le degré d'acidité ou d'alcalinité, la concentration des ions d'hydrogène. Ainsi donc, quand il est aidé par une forte acidité, dans un milieu dont le $p H$ est voisin de 2, ce ferment agirait comme pepsine, attaquant la molécule de protéine 
pour la dégrader finalement jusqu'au terme d'albumose et de peptone. En milieu neutre, légèrement acide ou légèrement alcalin, le ferment de la muqueuse stomacale ne provoquerait, au contraire, qu'une protéolyse légère, dont la coagulation serait finalement la conséquence et souvent la seule transformation apparente-

L'école dualiste, à laquelle les recherches les plus récentes semblent donner raison, combat la thèse de l'identité des ferments solubilisants et coagulants, et établit une distinction bien nette entre la chymosine et la pepsine. Pami les observations qui militent en faveur de cette thèse, nous ne rappelons ici que celles de FENGER, d'une part, et de TAUBER, d'autre part, tous deux auteurs américains. En partant de macérations de caillette de veau, FEnGER et TAUBeR réussirent, indépendamment l'un de l'autre, par des procédés différents de dissolution et de précipitation, à concentrer sur un résidu l'effet-présure tout en diminuant, sans cependant le faire disparaître complètement, l'effet-pepsine, c'est-à-dire l'effet solubilisant et protéolytique. TAUBER obtint en particulier de cette façon une présure dont l'activité, dépassant celle de toutes les présures préparées jusqu'alors, est si considérable qu'il suffit d'un gramme de celle-ci pour coaguler 4.550 litres de lait écrémé. Or, cette préparation, dont l'énergie peut eertainement être considérée comme un indice de pureté, possède une composition élémentaire et des propriétés qui s'écartent fortement de celle des préparations de pepsine les plus concentrées, en particulier la pepsine cristalline de Northrop. De ces constatations et d'autres encore qui les corroborent, nous nous croyons fondés à admettre que le ferment de la présure est un enzyme particulier, nettement distinct de celui de la pepsine.

Nous n'en énumérerons ici que les plus importantes propriétés : la présure est une protéine sulfurée; elle est soluble à l'eau et dans les solutions étendues de sel de cuisine et d'acide chlorhydrique. Elle donne une réaction de Millon négative, n'est pas coagulée par la chaleur et présente une grande résistance aux acides. Elle est rapidement altérée par la lumière, tout spécialement en présence d'eau et d'oxygène. Contrairement à la pepsine, dont l'action ne se développe qu'en milieu fortement acide, la présure, bien que favorisée aussi par une acidité modérée, agit déjà en milieu neutre ou même légèrement alcalin, jusqu'au $p \mathbf{H}$ supérieur limite de 7,4 ou 7,5. Disons aussi que si la pepsine s'attaque à toutes les protéines, quelles que soient leur origine et leur composition, et les amène finalement au stade d'albumoses ou de peptones solubles, l'action de la présure n'est ni aussi généralisée ni aussi profonde. Son rôle de ferment, plus spécifiquement orienté, ne se manifeste guère que sur la protéine principale du lait, la caséine, déterminant, par suite 
de certaines modifications chimíques et physiques, le phénomène de la coagulation du lait. Mais quelle est done cette action de la chymase sur la caséine? Je ne serais probablement pas compris de tout le monde si, voulant vous parler de ces modifications apportées par la présure, je ne vous disais pas d'abord ce que c'ést que la caséine.

La easéine est la protéine caractéristique de la sécrétion mammaire, voilà pour le côté physiologique. Les autres protéines du lait, albumine et globuline, déjà contenues dans le sang, ne sont pas le résultat d'un travail d'élaboration de la glande. L'édifice moléculaire de la caséine, protéine phosphorée, est extrêmement compliqué. Si nous nous en référons aux recherches les plus récentes, la caséine, une fois purifiée et débarrassée des matières minérales avec lesquelles elle se trouve associée, aurait un poids moléculaire voisin de 375.000. La dislocation de cette molécule, par l'action hydrolytique des acides, ne livre pas moins de 20 acides aminés, notamment tous ceux qui sont indispensables à l'édification des tissus musculaires du jeune mammifère. D'où déjà l'importance de cette protéine, que, du point de vue de notre alimentation et de celle des animaux, nous pourrons qualifier du nom de protéine complète.

Les progrès accomplis dans l'analyse des produits de décomposition hydrolytique de la caséine ont révélé, contrairement à ce qu'affirmaient les premiers chercheurs, que les fonctions acides dues aux groupements carboxyles $\mathrm{COOH}$ dépassent numériquement, par suite de la présence d'un grand nombre de molécules de diacides, les fonctions alcalines des groupes aminés $\mathrm{NH}^{2}$. Ainsi s'explique-t-on que la caséine, amphotère comme toutes les protéines, ait une acidité nettement caractérisée, lui donnant par exemple la faculté de s'approprier le métal d'un carbonate, tout en libérant l'acide carbonique, et le pouvoir de se réunir aux bases pour donner des sels. C'est sous cette forme salifiée de caséinate que nous rencontrons la caséine dans le lait. Peut-être, est-ce le moment de faire ici une première discrimination entre le lait de vache et le lait de nourrice : le premier, riche en chaux, contenant essentiellement la caséine comme caséinate de calcium ; le second, plus abondamment pourvu de sels alcalins, contenant, en partie tout au moins, la caséine comme caséinate de potasse et de soude. Si nous jugeons intéressant d'attirer l'attention sur ce point, c'est que la nature différente de ces caséinates nous aidera à comprendre tout à l'heure pourquoi ces deux types de lait se comportent différemment vis-à-vis de la présure.

Le poids moléculaire extrêmement élevé de la caséine et de ses sels, les caséinates, est certainement responsable de l'aspect col- 
loïdal que présentent ces composants du lait. Le caséinate de calcium du lait de vache existe en effet sous la forme de particules, de micelles, visibles sous I'ultra-microscope et dont la dimension moyenne doit être assez voisine de 30 millionièmes de millimètre. Si nous nous en rapportons à la classification la plus couramment utilisée des matières présentant l'état colloïdal, nous sommes amenés à dire que le caséinate appartient au groupe des colloïdes hydrophiles ou lyophiles, qui ont une grande affinité pour l'eau et qui doivent à cela une certaine stabilité, une certaine insensibilité à l'action floculente des électrolytes. Quand ils précipitent de leurs solutions, ces colloïdes hydrophiles donnent généralement un coagulum volumineux enrobant une très grande quantité de liquide. Tout autrement se comportent les colloïdes dits hydrophobes, lyophobes, dont il existe quantité d'exemples parmi les matières minérales.

Il est intéressant de noter encore que le caséinate de chaux le fait a été prouvé par de nombreux travaux et d'une manière très concordante - se trouve associé à des micelles plus grossières de phosphate de chaux. Nous ne sommes pas encore absolument au clair sur la nature des liaisons qui groupent en une sorte de complexe dans le lait le caséinate et le phosphate de chaux. Ce que l'on peut dire dans l'état actuel de nos connaissances sur cette question, e'est qu'il faut renoncer à faire intervenir l'influence des valences primaires, des forces chimiques. Par ultra-centrifugation, il a été possible en effet de fractionner ce complexe en des échantillons contenant des proportions très différentes de phosphate de chaux. Tout se passe comme si ces micelles de complexe caséinatephosphate, dissociées par la simple influence d'une action purement physique, étaient constituées en réalité par un noyau relativement grossier de phosphate autour duquel seraient venues se rassembler des particules beaucoup plus petites de caséinate. Le phosphate triealcique épouserait alors les propriétés du colloïde qui l'enveloppe, perdrait son caractère de colloïde instable, facilement coagulable par les électrolytes. En d'autres termes, nous aurions affaire ici au cas bien connu de la protection d'un colloïde hydrophobe par un colloïde hydrophile. Quand dans la suite de notre exposé nous parlerons de la easéine, comme on la rencontre dans le lait, il s'agira donc en réalité de complexe phosphate-caséinate de chaux.

On a beaucoup discuté sur le point de savoir si la caséine était une substance homogène, et si elle était identique dans tous les laits, notamment dans les laits livrés par les différentes espèces mammifères. La controverse n'est pas terminée. Les contributions scientifiques apportées récemment en vue de résoudre ce problème ne sont pas de nature à fournir des certitudes quant à l'homogénéité ou 
la non-homogénéité de la caséine et quant à la constance ou à la variabilité de sa composition. Par leurs recherches spectroscopiques, portant sur des caséines purifiées de diverses origines, ADBERHALDEN et RossIer parviennent à la conelusion qu'il existe une concordance très étroite, peut-être même une similitude complète dans la structure chimique intime des caséines des divers laits étudiés. Cherbulliez et Schneider disent, au contraire, avoir séparé de la caséine du lait de vache par le jeu de dissolutions et de précipitations qui n'ébranleraient pas l'édifice moléculaire original quatre protéines distinctes par leur composition et leurs propriétés. L'une d'elles, la plus abondante, la easéine $\mathrm{L}^{\prime \prime}$, insoluble dans les solutions de chlorure d'ammonium, précipitée d'une solution de soude caustique au $p \mathrm{H}$ 4,8 par un apport d'acétone, serait la plus sensible à l'action de la présure. C'est d'elle que la caséine tiendrait ses propriétés essentielles. Cherbulliez pense pouvoir attribuer à l'inégale distribution de cette caséine $\mathrm{L}^{\prime \prime}$ dans les caséines brutes, l'aptitude différente des laits à coaguler sous l'action de la présure. Sans vouloir contester la valeur des observations sur lesquelles est fondée cette dernière hypothèse, nous remarquerons déjà qu'il n'est pas forcément nécessaire de recourir à celle-ci pour expliquer la réaction paresseuse de certains laits vis-à-vis de la présure.

En dépit de sa simplicité apparente, le phénomène de la coagulation du lait par la présure est d'une extrême complexité. Les contingences qui l'entourent et peuvent en modifier la marche sont nombreuses. La concentration de la caséine dans le lait, la quantité et la force de la présure mise en œuvre, la réaction du milieu, la richesse du sérum en sels alcalins et alcalino-terreux, les proportions d'albumine et de globuline, le taux de matières grasses, la présence ou l'absence de gaz carbonique, voilà autant de facteurs qui exercent leur influence, tantôt ajoutant leur action, tantôt se contrariant. Parce qu'on n'a pas su d'emblée mettre en évidence le rôle propre à chacun de ces facteurs et le dégager des actions voisines, l'interprétation des faits observés a souvent été malheureuse. Peu de phénomènes ont fait surgir autant d'hypothèses que celui de la coagulation du lait. En 1839 déjà, TREMY hasarde une première explication; il croit pouvoir attribuer la formation du caillé à la transformation du lactose en acide lactique. Cela revenait à assimiler la coagulation par la présure à la coagulation résultant de l'acidification naturelle du lait. On sait aujourd'hui que ces deux phénomènes de la coagulation par les acides et par la présure ont peu de points communs et que leur apparente similitude cache des transformations d'ordre chimique bien différentes. C'est encore le même point de vue qui est soutenu quelques années plus tard par LIEBIG et par SoxhLET. 
Depuis Limbig et jusqu'à aujourd'hui les théories qui ont vu le jour, peuvent être rangées, si on veut souligner l'orientation des idées qui ont présidé à leur élaboration, en deux groupes : les théories physiques, à l'éclosion desquelles a puissamment eontribué le récent développement de la chimie colloïdale, et les théories chimiques, qui admettent comme point de départ tout au moins une action chimique de la présure sur la caséine. Nous n'attendrons pas davantage pour dire que les théories physiques, dont nous allons. nous oceuper d'abord, ont eu peu de succès, soit qu'elles aient été mises en échec par des recherches ultérieures, soit qu'elles n'aient pu rendre compte que d'une partie des faits observés. Quelquesunes n'ont même probablement jamais satisfait que leurs auteurs.

Alexander, remarquant déjà que la caséine - nous parlons évidemment ici du complexe naturel caséinate-phosphate de chaux - n'est pas en solution vraie dans le lait, mais en dispersion micellaire, attribue la stabilité de ces solutions colloïdales de caséine au rôle protecteur de l'albumine. Pour lui, la présure détruirait eet effet protecteur et, sans modifier d'aucune façon la structure chimique de la easéine, provoquerait simplement l'union et la soudure des micelles, puis finalement la formation d'un coagulum ou gel. C'est une façon de voir. Le malheur est que le easéinate de chaux n'a pas besoin d'albumine pour se maintenir en dispersion, $q$ u'une addition d'albumine n'accroît pás la stabilité de ses suspensions et qu'enfin, il n'a jamais pu être donné la preuve que la présure exerçât une action queleonque sưr l'albumine. Il a bien fallu abandonner cette explication et rechereher ailleurs l'origine de l'action de lab.

En s'appuyant sur des observations qui n'ont d'ailleurs rien à voir avec les faits qu'elles sont censées expliquer, ScHRxver formule l'opinion que les matériaux constituant le caillé préexistent déjà dans le lait, mais que leur agrégation en est empêchée par adsorption depuis le sérum de certaines substances protectrices. La fonction de la présure serait ici de libérer la surface des micelles de ces produits d'adsorption. Il n'est pas nécessaire d'insister sur tout ce qu'a d'imprécis une telle théorie.

LoEWENHART, qui raisonne également en physicien, estime que la présure provoque, au simple contact avec le lait, une première union des micelles de caséine et détermine ainsi la formation d'un colloïde moins parfaitement dispersé, très instable, coagulant facilement sous l'influence des sels eontenus dans le sérum. On est peu enclin à accorder un large crédit à cette hypothèse si l'on songe que plusieurs expérimentateurs, notamment les allemands LAQUEUR et Michoff, croient avoir noté précisément le contraire et n'hésitent pas à fonder sur ces observations diamétralement opposées l'expli- 
cation du pouvoir coagulant de la présure. Les théories de DuoLAux et Cosmovici suivant lesquelles la coagulation serait un simple phénomène d'adhésion moléculaire déclenché par la présure ne résiste pas davantage à l'épreuve d'une argumentation scientifique rigoureuse ou à celle de l'expérimentation. Nous n'avons mentionné que les plus connues de ces théories physiques de l'emprésurage. Il en existe d'autres, qui font intervenir, cette fois, l'influence des charges électriques portées par les colloïdes de la présure et même certaines vibrations engendrées par le ferment. Tout ceci est assez imprécis, assez mystérieux. Ce que j'en ai dit suffira, je pense, à montrer que nous ne nous trouvons pas sur un terrain vraiment solide. A toutes ces hypothèses, il faut adresser le même reproche, qu'elles s'attachent trop à la forme et négligent le fond. Ce n'est pas sans danger qu'on s'écarte du côté chimique des choses pour ne vouloir juger celles-ci que par leur aspect, nous dirons comme Porcher leur habit colloïdal, On s'expose à des explications qui séduisent parfois par leur simplicité, mais qui au fond n'expliquent rien et ne réussissent qu'à paralyser la recherche et à retarder le progrès. C'est en effet à cette tournure d'esprit qu'on doit l'abandon, disons plutôt le relèguement, d'une conception chimique de l'action de la présure qui s'était pourtant révélée fructueuse dès le début, conception qu'il a fallu pourtant reprendre à eause de l'insuffisance des explications physico-colloïdales dont nous avons parlé. Nous faisons allusion à la théorie d'HAMMERSTEN, vieille de plus de 50 ans déjà, que les travaux les plus récents ont vérifiée et jus tifiée, tout au moins dans ses lignes essentielles. HAMMERSTEN prétendit le premier que la présure, à laquelle il donnait déjà le nom de chymosine, modifie la structure moléculaire du caséinate, le dédouble en deux produits d'importance pondérale très différente, à savoir une protéose soluble ne représentant guère que le $4 \%$ du poids de la molécule attaquée et un résidu solide qui constituera plus tard la masse du coagulum et auquel il donna le nom de paracaséinate. En somme, le paracaséinate est le sel de la caséine modifiée par le ferment du lab. Tout ceci équivaut à dire que la présure exeree sur la caséine ou le caséinate une action protéolytique, peu profonde mais nettement définie et orientée, sans laquelle la coagulation ne pourrait pas avoir lieu. Le même point de vue est soutenu quelques années plus tard par ARTHus et PAGÈs. Les travaux de ces deux auteurs, qui donnent une lumineuse confirmation des idées d'HAMmersten, soulignent déjà avec insistance les faits, aujourd'hui reconnus exacts, que la coagulation du lait par le ferment présure comprend deux phénomènes successifs distincts : $1^{0}$ la transformation chimique de la caséine en paracaséine et $2^{\circ}$ la précipitation des particules de paracaséine pour former le coagulum. 
ARthus et PAGÈS reprenaient done à leur compte cette idée fondaimentale que le lab n'est pas l'agent direct de la coagulation, mais l'agent indirect de celle-ci, par les modifieations chimiques de la easéine qu'il détermine, et qu'à ce titre, il peut prendre place à côté de la pepsine, de la trypsine et autres ferments des matières albuminoïdes.

Voici quelques observations expérimentales dans lesquelles nous voulons voir la preuve que la présure n'entraîne pas seulement dans le lait des transformations d'ordre physique, mais un véritable clivage chimique de la molécule de caséine :

1. La protéose soluble contenue dans le sérum d'emprésurage, signalée pour la première fois par HAMMERSTEN, a été observée par de nombreux expérimentateurs. ARthus et PAGÈs en font mention et lui donnent le nom d'hémi-caséinogène. Les travaux de Coster, Fuld, Muller, Petry, Spiro, Slocotzoff, Schmidt, Nielsen, etc. la mettent tous en évidence. Porcher lui-même en montre la formation en faisant agir la présure non plus sur le lait, mais sur le complexe caséinate-phosphate de chaux obtenu par synthèse. Nous avons eu l'oceasion d'en noter nous-mêmes l'apparition.

2. Une autre preuve de cet effet chimique de la présure sur la easéine repose sur l'étude comparée des propriétés de la caséine et de la paracaséine. Sans doute, toutes les tentatives faites pour établir une distinction chimique entre ces deux protéines n'ont pas toujours été couronnées de succès. Sans doute, l'échec essuyé a-t-il pu laisser croire à l'identité de ces substances. Des recherches plus récentes nous font croire plutôt que cet insuccès résulte probablement du manque de sensibilité de certaines méthodes physicochimiques utilisées et de leur inaptitude à déceler une transformation qui n'est probablement pas un bouleversement moléculaire mais un simple réarrangement. Les méthodes biochimiques se sont révélées plus délicates. Les résultats qu'elles fournissent ne paraissent laisser auoun doute sur la spécificité de constitution de la caséine et de la paracaséine. Nous ne citerons ici que les travaux de Nicolas. Cet auteur étudie le pouvoir antigène de ces deux substances en recherchant de quelle façon se comportent les solutions de leurs sels sodiques à l'égard des antisérums obtenus de lapins qui ont été traités au moyen d'injections répétées de l'un ou de I'autre de ces deux corps. Nicolas n'éprouve pas de peine à montrer que cette méthode des sérums précipitants permet une différenciation certaine de la caséine et de la paracaséine. Il est conduit à la conclusion intéressante que si ces deux protéines sont certainement apparentées l'une à l'autre, d'abord par leur composition élémentaire, par l'identité de la plupart de leurs constantes physico- 
chimiques et même par certains earactères immunologiques communs, leur façon différente de se comporter vis-à-vis des précipitines traduit une différence certaine dans leur nature chimique.

La non-identité de la caséine et de la paracaséine a pu être établie aussi par l'étude comparée de quelques-unes de leurs propriétés chimiques, notamment de leur pouvoir de combinaison pour les bases et les acides. Dans un travail que nous avons effectué dans un laboratoire américain, et par lequel nous voulions rechercher si la easéine et la paracaséine forment avec les bases et les acides des combinaisons chimiques suivant la loi d'action de masse ou, au contraire, des combinaisons d'adsorption d'après les relations de FreUNDLICH et de LANGMUIR, nous étions amené à conclure que les quantités d'ammoniaque et d'acide chlorhydrique fixées stoechiométriquement par ces substances ne sont pas exactement les mêmes. La paracaséine fixe plus d'acide et plus de base que la caséine. Il faut voir là nécessairement le résultat des modifications moléculaires engendrées par la présure.

Si tout ce que nous venons de signaler suffit à établir que la présure exerce une action chimique certaine sur la caséine, il est plus difficile d'indiquer, en dehors des quelques renseignements que nous avons pu en donner, en quoi consiste exactement le dédoublement provoqué par la présure et en quoi la molécule de caséine se distingue de celle de la paracaséine. Oh ! sans doute, les hypothèses ne manquent pas. Quelques-unes mêmes sont ingénieuses et reposent sur des argumentations et des rapprochements séduisants. Les unes et les autres manquent d'une base solide expérimentale.

Bang, Palmer et Richardson considèrent que le lab détermine une hydrolyse de la caséine comparable à celle qui dédouble le maltose en glucose. La molécule de caséine se transformerait donc en deux molécules de paracaséine, substance nouvelle possédant la propriété de coaguler plus facilement. MELLanby assimile cette transformation de la caséine au phénomène de dénaturation que produit la chaleur sur l'albumine du blanc d'œuf. L'ennui est que nous sommes fort mal renseigné sur la nature des modifications chimiques que subit alors la molécule d'albumine. Plus récemment, BEAU se donna beaucoup de peine pour montrer que la paracaséine pourrait être un simple produit de polymérisation de la caséine, dans la formation duquel la caséine participerait comme catalyte. BEAU voit une analogie entre la formation de la paracaséine sous l'action du lab et les phénomènes de polymérisation qui donnent lieu à la production de la bakélite quand on chauffe du phénol en présence de formol et d'ammoniaque. Indépendamment du reproche 
que l'on peut faire à BEAU de ne pas apporter de preuves expérimentales justifiant son point de vue, il serait aisé encore de lui opposer plusieurs objections. Le rôle chimique que BEAU fait jouer au phosphate de chaux comme plastifiant et comme élément de condensation des molécules de caséinate, est en contradiction avec les faits montrant que caséinate de chaux et phosphate de chaux, bien que réunis dans les mêmes micelles, sont chimiquement distinets.

Autant avouer, je crois, que dans l'état actuel de nos connaissances, nous ne savons paś, même d'une façon approchée, en quoi la molécule de paracaséinate se distingue de celle du caséinate dont elle est issue. Ce que nous pouvons dire, par contre, c'est que ces modifications chimiques retentissent nettement sur les propriétés physiques, colloïdales de la substance. La caséine étant un colloïde hydrophyle ou relativement hydrophyle, - car tout est une question de degré, - la paracaséine est un corps nettement moins hydraté, possédant déjà eertains caractères des colloïdes hydrophobes. Les preuves ne manquent pas à l'appui de cette affirmation : tout de suite après son mélange au lait et avant que la coagulation ait pu commencer, la présure détermine une diminution sensible de la viscosité. Autre conséquence d'une hydratation diminuée, les micelles sont plus facilement visibles sous l'ultra-microscope et leur mouvement brownien plus rapide. Enfin, ces micelles de paracaséinates sont moins stables, beaucoup plus sensibles à l'action floculente des électrolytes que celles des suspensions originelles de caséinate. Nous touchons là à l'explication du phénomène de la coagulation, qui traduit pour l'observateur superficiel toute l'action de la présure, mais qui n'en constitue en réalité que la manifestation secondaire et indirecte. Nous avons mentionné qu'Hammersten, puis ARThus et PAgìs avaient déjà discriminé dans le phénomène de l'emprésurage des laits deux phases, deux temps successifs distincts : $1^{0}$ l'action chimique de la présure sur le caséinate et sa conversion en paracaséinate, 20 la coagulation du paracaséinate. C'est à PORCHER, auteur français dont le nom est attaché à de nombreuses études importantes sur le lait, que revient le mérite d'avoir donné à la théorie d'HAMMERSTEN une consécration expérimentale éclatante tout en la précisant et la complétant sur un point fondamental : PoRcher montra que la coagulation du paracaséinate devrait être rapportée à certains éléments cristalloïdaux contenus en solution dans le lait, notamment à la chaux contenue surtout sous forme de phosphate monocalcique. Le lait qui a été dyalisé et débarrassé par ce moyen des éléments salins solubles qu'il contenait ne coagule par la présure que si on lui rajoute après coup les sels de chaux dont l'opération l'a dépouillé. Sans addition de ces sels, 
aucune trace de floculation, aucune prise en masse. Cependant, la formation d'une protéose soluble, d'une part, l'apparition, d'autre part, de tous les signes trahissant la transformation du caséinate en un colloïde plus éloigné par sa nature des colloïdes hydrophiles et plus près des colloïdes hydrophobes, en imminence de précipitation, sont là pour nous montrer que le lab a développé son action chimique habituelle, mais que l'absence de matières salines a simplement interdit le deuxième temps de l'emprésurage, la réunion des micelles de paracaséinate. Les mêmes observations peuvent être faites lorsqu'on éprouve la présure sur un lait écrémé, filtré sur bougies de poreelaine et dont le résidu a été dispersé dans de l'eau distillée. Ajoutons que les édifices synthétiques, easéinatephosphate de chaux, se comportent d'une façon absolument pareille et que leur prise en masse sous l'action de la présure est subordonnée à l'addition d'une quantité minimum de sels de chaux soluble. Ce que nous venons de dire fait certainement comprendre pourquoi certains laits de coagulation paresseuse, naturellement peu propice à la fabrication de fromage, tranchent plus facilement après une adjonction de chlorure de calcium et pourquoi la cuisson du lait ou simplement sa pasteurisation à une température voisine de $70^{\circ} \mathrm{C}$., qui insolubilise \pm complètement le phosphate de chaux, diminue ou détruit l'aptitude du lait à coaguler sous l'action de la présure.

Si l'on ne prête à la coagulation du lait par la présure qu'une attention distraite, on ne percevra probablement que l'aspect final de ee phénomène. Celui-ci se traduit simplement pour nous par la prise en masse du liquide, par sa gélification, par la formation d'un bloc compact et élastique, se contractant peu à peu et laissant exsuder par synérèse le sérum qu’il emprisonnait. Si vous vous employez, par contre, à noter, de seconde en seconde, les transformations qui conduisent à cette solidification du lait, il ne vous échappera pas que ce liquide passe durant la coagulation par un certain nombre de stades, toujours les mêmes, se succédant dans un même ordre. Le lait écrémé qui vient d'être additionné de présure commence par perdre ses reflets bleutés; on le voit qui devient plus blanc et visiblement plus opaque; plus tard, on surprend un épaississement du lait, auquel succède bientôt la résolution du liquide en flocons ; enfin, ces flocons s'unissent pour former la masse compacte de coagulum ou caillé. Si ce coagulum volumineux, à structure réticulée, que d'aucuns ont comparé à une éponge gonflée de sérum et dont les propriétés présentent plus qu'une analogie avec les gelées décrites et étudiées par FoDor, est en somme, dans les conditions d'un emprésurage normal, le point d'aboutissement de la coagulation du lait de vache, il n'est pas vrai que tous les laits puissent prendre 
finalement, en coagulant, cette forme gélifiée. Certains résistent même à toute tentative faite dans le but d'obtenir leur prise en masse. La forme floculente de la coagulation est parfois la seule possible, la seule réalisable. Des cas existent où à la suite d'une addition de présure les micelles de caséine parviennent bien à se réunir en flocons, mais où ces flocons ne se soudent plus pour former un caillé. Le type le plus eonnu de ces laits à coagulation floconneuse est le lait de nourrice. De nombreux travaux ont été entrepris pour trouver les raisons de cette attitude spéciale vis-à-vis de la présure. On a voulu voir dans cette inertie relative du lait de femme à l'action de la présure une preuve de la composition spécifique de sa caséine. Les recherches les plus récentes n'ont pas justifié ce point de vue. Si l'état de nos connaissances ne nous permet pas de croire à l'existence d'une caséine chimiquement définie et la même pour tous les laits, - les travaux de LINDERSTrom LANG, puis de Cherbulliez, nous préparent plutôt à admettre le contraire, tout porte à croire qu'il ne faut pas chercher la cause principale de cette coagulation floconneuse du lait de nourrice dans une composition spécifique de sa caséine, mais dans les circonstances particulières qui entourent ce composant du lait : plus grand degré de dilution de la caséine ; équilibre salin différent, résultant de la présenee d'une plus forte concentration de sels alealins ; teneur plus élevée en protéine protectrice (globuline); réaction légèrement alcaline, assez voisine du $p H$ supérieur limite d'action de la présure.

Nous voici conduit au chapitre final de notre thème : l'examen des facteurs sous la dépendance desquels se trouve la réaction des laits à la présure. Disons d'abord que la littérature sur ce sujet, extrêmement volumineuse, ne nous renseigne malheureusement pas avec précision et avec netteté. Les contradictions sont nombreuses. Une certaine confusion règne encore. A cela, il y a probablement deux raisons. La première, c'est que dans l'étude de la coagulation du lait par la présure et des facteurs qui influent sur la marche du phénomène, beaucoup d'auteurs n'ont pas distingué comme il l'aurait fallu les deux stades successifs : $1^{0}$ la transformation du caséinate en paracaséinate par l'action chimique du ferment, $2^{\circ}$ la précipitation du paracaséinate par les sels de chaux de la solution. L'autre raison réside dans le fait que ces facteurs sont nombreux, $q u$ 'ils agissent en étroite interdépendance, enchevêtrant souvent leur action, et qu'il est parfois très malaisé de disséquer l'action résultante pour définir le rôle propre, particulier à chacun d'eux.

L'action diastasique chimique de la présure, tout au moins de celle qu'on utilise le plus généralement et qui provient de l'estomac de veau, se poursuit surtout rapidement à la température du corps de l'animal, température voisine de $40^{\circ}$. Elle est détruite en quel- 
ques minutes quand on la chauffe jusqu'à $60-65^{\circ}$. Quand on maintient la température du lait en dessous de $10^{\circ}$, on ne constate plus de coagulation. Le caillé qui est ferme lorsque, pour l'emprésurage, le lait a été chauffé à $40^{\circ}$, prend une consistance d'autant plus molle qu'on s'écarte davantage de cette température optimale. La réaction du lait est un autre facteur important de l'emprésurage. C'est en milieu légèrement acide, entre les $p H$ et 6,4 , que le travail chimique du ferment est le plus rapide et le plus complet. Lorsque le $p \mathrm{H}$ s'accroit, donc quand la réaction devient neutre ou alcaline, la transformation de la caséine en paracaséine se ralentit. Elle cesse au delà des $p H$ 7,4 ou 7,5. Pour cette raison, toute addition d'alcali au lait se traduit par un retard de la coagulation, la rapidité de ce phénomène dépendant nécessairement de la vitesse avec laquelle se forme la paracaséine sous l'action de la présure. Souvent aussi ces coagulations paresseuses, dues à l'alcalinité, présentent les caractères de coagulation incomplète, ne dépassant pas le stade de la formation de flocons. D'où l'on voit déjà que dans la recherche des causes possibles d'une coagulation défectueuse, il est indiqué de voir s'il ne faut pas incriminer une réaction défavorable. Il est bien connu, par exemple, que certains laits pathologiques obtenus d'animaux atteints d'une affection mammaire, montrent un défaut caractérisé de sensibilité à la présure, défaut fort gênant quand le lait est destiné à la fromagerie. L'étude de ces laits malades montre que ce défaut est en relation avec une alcalinité anormale. La même observation peut être faite avec le lait de nourrice, dont le $p \mathbf{H}(7,2)$ est assez rapproché du $p H$ supérieur limite au delà duquel la présure cesse d'exercer une action chimique sur la caséine. Il faut toutefois préciser dès maintenant que l'alcalinité n'est pas le seul facteur responsable de la mauvaise coagulation de ces laits. Leur plus grande richesse en sels de métaux monovalents, soude et potasse, et leur teneur élevée en globuline jouent encore un rôle important. Cependant, nous remarquerons que ces deux facteurs n'agissent plus, cette fois, sur le pouvoir ferment du lab, mais sur le deuxième temps de l'emprésurage : l'agglomération des micelles de paracaséinate.

De nombreux expérimentateurs se sont occupés de l'influence des sels du lait sur l'action de la présure. Duclaux, dans ses importants mémoires sur le lait, examine déjà tour à tour le rôle des divers sels alcalins et alcalino-terreux et conclut que si l'action de ces sels est extrêmement variée, on peut, malgré cela, formuler la règle que les métaux alcalins, sodium et potassium, rendent la caséine plus difficilement précipitable par la présure. Cet antagonisme entre les sels alcalino-terreux de chaux et de magnésie, que nous avons rendus responsables de la coagulation des micelles de paracaséinate, 
et les sels alcalins de soude et de potasse, qui la contrarient, a été mis en évidence par quantité de travaux. C'est ce qu'ont voulu dire certains auteurs quand ils affirment que les métaux alcalins sont pour la caséine des éléments peptisants, stabilisants, tandis qu'ils qualifient la chaux et la magnésie d'éléments coagulants. Pouvonsnous donner une explication ? Si nous nous en référions à la règle par laquelle Schulze a montré la relation existant entre la valence des métaux et leur pouvoir coagulant sur les solutions ou suspensions d'un colloïde négatif, comme le paracaséinate, nous devrions attribuer aux sels de soude et de potasse eux-mêmes la faculté de coaguler. Celle-ci serait simplement, toutes autres conditions restant inchangées, moins considérable que celles de solutions équivalentes de chaux ou de magnésie. Au lieu de cela, nous avons dit que les sels de soude et de potasse exercent une action nettement inhibitrice sur la coagulation par la présure. Où faut-il en recherchêr la cause ? Celle-ci réside certainement dans les relations d'équilibre chimique existant entre le caséinate et la solution. En présence d'un sérum riche en sels alcalins, le caséinate de chaux se convertit partiellement en caséinate de soude et de potasse, dont le produit de transformation par la présure, le paracaséinate, n'a plus exactement les mêmes propriétés. Les observations ne manquent pas qui sont autant d'indices ou de preuves attestant que les micelles de ce paracaséinate alcalin sont plus hydrophiles que celles du caséinate de chaux correspondant. Cette plus grande affinité des micelles pour l'eau est une cause de stabilité ; elle permet au colloïde de résister plus facilement au pouvoir coagulant des sels de la solution. Il faudra désormais une concentration plus considérable de chaux pour déterminer la coagulation et si celle-ci parvient à former un caillé, ce dernier reflétera par sa nature molle, inconsistante, le earactère hydrophile des micelles qui lui ont donné naissance. C'est un caillé de ce genre que l'on obtient lorsqu'on emprésure certains laits malades. On ne songera pas à s'en étonner si l'on sait que le fonctionnement anormal de la glande lactifère conduit à des modifications de la composition du lait, qui sont orientées presque toujours dans la même direction : disparition plus ou moins complète de lactose et son remplacement pour rétablir l'équilibre moléculaire et osmotique par du chlorure de sodium. La réaction du lait à la présure change simplement parce que l'édifice salin s'est modifié en faveur d'une plus forte concentration de sels alealins.

Enfin, si nous nous souvenons que le lait de nourrice renferme proportionnellement davantage de $K$ et autres éléments gênant la formation d'un coagulum compact, que le lait de vache, nous comprendrons sans peine qu'on ait pu recommander, en diététique 
infantile, d'enrichir le lait de vache en sels alcalins, par l'addition d'une certaine charge de citrate de soude ; on se proposait ainsi de rendre le lait de vache plus semblable à celui de la mère et d'en faciliter la digestion.

A côté de l'alcalinité, qui paralyse l'action chimique de la présure et des sels alcalins de soude et de potasse, qui déterminent la formation d'un paracaséinate aux micelles hydrophiles et stables, un troisième facteur peut venir contrecarrer encore la marche de la coagulation par la présure : e'est la présence dans le lait d'une certaine quantité de globuline, jouant le rôle de colloïdes protecteurs. La globuline se rencontre normalement déjà dans le lait de vache, mais en faible quantité seulement, si ce lait a été livré par une mamelle saine. L'analyse en décèle déjà une proportion plus importante dans les laits pathologiques, dans les laits de rétention, c'est-à-dire ceux qui ont séjourné trop longtemps dans la mamelle et dans le colostrum. La forte teneur en globuline est aussi un trait particulier de la composition du lait de femme et d'autres laits encore qui réagissent mal à la présure et ne donnent qu'une coagulation floconneuse. Comment concevoir cette action protectrice de la globuline? Nous en demanderons l'explication à un chapitre bien connu de la chimie colloïdale, celui qui traite de l'action des colloïdes, les uns sur les autres. Certains colloides hydrophiles, stables - la globuline est au nombre de ces derniers - peuvent être adsorbés de leurs solutions par des colloïdes hydrophobes, en suspension plus grossière, auxquels ils communiquent leurs propriétés. De nombreuses observations montrent en effet que la globuline de lait tend à se concentrer à la surface des micelles de caséinate et paracaséinate. Nous sommes donc fondés à admettre qu'en épousant la nature hydrophile de la globuline qui les recouvre, les particules de paracaséinate peuvent échapper dorénavant à l'action coagulante des sels se trouvant dans le sérum.

Réaction différente des laits, inégale distribution des sels de soude ou de potasse peptisants et des sels de ehaux coagulants, teneur variable en colloïde protecteur (globuline), telles sont les trois raisons principales pour lesquelles tous les laits ne réagissent pas avec la même rapidité et de la même façon, à l'action de la présure.

La coagulation par la présure est influencée encore par la concentration de la caséine, par celle du ferment, par la richesse du lait en matière grasse, par sa teneur en gaz carbonique. L'heure est assez avancée pour que je n'aie pas la tentation de discuter le rôle de chacun de ces facteurs. J'aime mieux, en terminant maintenant mon exposé, mériter le reproche d'être incomplet que d'encourir celui d'avoir été trop long. Je n'avais d'ailleurs pas 
d'autre prétention que de montrer les aspects principaux du phénomène de la coagulation du lait par la présure.

\title{
ÉTUDE DE L'APPRÉCIATION DE LA QUALITÉ DES CASÉINES LACTIQUES
}

\author{
par \\ JEAN PIEN \\ Ingénieur chimiste I. C. R. \\ Docteur ès sciences \\ et S. HERSCHDCERFER \\ Docteur ès sciences (Vienne)
}

Directeur des laboratoires des "Fermiers Rúunis "

\section{ROBERT MARTIN}

Ingénieur-Chimiste I. C. R.

des Laboratoires des "Fermiers Réunis »

\section{(Fin.)}

Le but que nous nous sommes proposé dans cette étude est l'appréciation de la qualité des caséines lactiques.

Dans notre premier article nous avons montré que tous les aecidents possibles dans la fabrication de ces produits pouvaient se traduire sur le plan analytique par l'apparition dans la caséine :

$1^{0}$ de protéines dégradées ;

$2^{\circ}$ d'éléments étrangers minéraux ou organiques fixés chimiquement ;

$3^{\circ}$ d'éléments étrangers minéraux ou organiques simplement mélangés à la caséine.

Le contrôle analytique des caséines en vue du dépistage des accidents de fabrication devrait done être capable de mettre en évidence ces divers éléments.

Quels sont ces éléments ?

$1^{0}$ Les diverses formes d'azote soluble provenant de la dégradation des protéines;

$2^{\circ}$ Eléments étrangers organiques : acide lactique, lactose ;

a) Mélangés à la caséine : acide lactique extérieur, lactose ;

b) Fixés à la caséine : lactate de caséine ;

$3^{\circ}$ Eléments étrangers minéraux : les sels de calcium, les phosphates, etc ;

a) Mélangés à la caséine : lactates de chaux, phosphates de chaux, etc.; etc.

b) Fixés à la caséine : caséinate, phosphocaséinate de chaux,

La détermination des formes d'azote soluble a été étudiée. 